\title{
SEMIÓTICA DE LA TRADUCCIÓN, TRADUCCIÓN DE LA SEMIÓTICA
}

\author{
Peeter Torop \\ Universidad de Tartu, Estonia \\ (Traducción del ruso de Rafael Guzmán)
}

En el desarrollo de la teoría de la traducción y de la semiótica existe una cierta analogía. La teoría de la traducción, como ciencia interdisciplinar relativamente joven, se encuentra en un estadio de sinonimia científica, por la igualdad entre los diferentes trabajos o por su diferencia tan sólo en el nivel del metalenguaje empleado, llegando incluso a la compilación y al plagio. Este estado lleva a eminentes teóricos de la traducción a una sensación de estancamiento. Se puede hablar de dos reacciones principales ante esta situación. En la base de la primera reacción se encuentra el deseo de alcanzar una metodología comprensible, una unidad de autoconsciencia disciplinar. La segunda reacción está relacionada con la aspiración de elevar la comprensión mutua en el marco de una ciencia, de un monolingüismo disciplinar.

Como ejemplo de la primera reacción puede servir el teórico de la traducción alemán $\mathrm{W}$. Wilss, que ve como salida del estancamiento la 
ampliación de la perspectiva metodológica. Considera como una posibilidad la explicitación máxima del análisis del proceso de la traducción, que incorpora el estudio de problemas de traducción comunes, independientes de un par concreto de lenguas (competence in translation), a los problemas prácticos, que emanan de una pareja concreta de lenguas (performance in translation) ${ }^{1}$. Por tanto, distingue una ciencia de la traducción prospectiva (dificultades de la traducción, enseñanza de los traductores) y una ciencia de la traducción retrospectiva (análisis de errores, crítica de la traducción) ${ }^{2}$.

Como ejemplo de la segunda reacción puede servir el teórico de la traducción húngaro $\mathrm{D}$. Radó, que considera la reevaluación de los trabajos científicos ya existentes como lo fundamentalal. En su opinión, la teoría de la traducción necesita ante todo elaborar una bibliografía completa de las investigaciones dispersas y la consecuente generalización y síntesis de los trabajos escritos desde perspectivas científicas totalmente diferentes ${ }^{3}$.

Ambos planteamientos suponen la aparición de discusiones metodológicas. $\mathrm{Y}$ es comprensible el temor de que estas discusiones no sean suficientemente productivas si no se alcanza una comprensión mutua en la utilización simultánea de metalenguajes diferentes e incluso herméticos. Por eso se escuchan llamadas para que las discusiones se limiten a un metalenguaje científico general y más o menos comprensible ${ }^{4}$. Por supuesto, esto es más difícil por cuanto en la teoría de la traducción no existe todavía un metalenguaje propiamente tradicional y estándar.

Si las dificultades metalingüísticas de la teoría de la traducción surgen de su interdisciplinariedad, las dificultades de la semiótica, como ciencia disciplinarmente más monolingüe, surgen de la ampliación de la problemática estudiada con ayuda de los métodos semióticos y del metalenguaje semiótico. Continuando el esquema de desarrollo de la semiótica, propuesto por U. Eco, y que incluye cuatro etapas (los años 60 como aproximación de la semiótica a la lingüística; finales de los 60 como una retirada gradual de los modelos lingüísticos; principios de

1 Wilss, W. The Science of Tanslation. Problems and Methods. Tübingen: Gunter Narr Verlag, 1982, 14-15.

2 Wilss, W. Op. cit., 159.

3 Radó. D. Basic Principles and Organized Reseach of the History, Theory and History of Theory of Translation. Der Obersetzer und seine Stellung in der Öffentlichkeit. Wien: Wilhelm Braumüller, 1985, 305.

4 Bassnett-McGuire, S. Translation Studies. London, New York: 1980, pág. 134. 
los 70 como preponderancia del interés por el engendramiento de textos, por el proceso de semiotización; mediados de los 70 como paso del estudio del engendramiento al estudio del consumo, a la recepción de los textos), J. Monaco considera una quinta etapa la institucionalización académica de la semiótica (the academic establishment of semiotics ${ }^{5}$, que caracteriza ante todo a la tradición anglo-americana en el umbral de los años 80 . Constata que la moda por la semiótica la hace peligrosa, los medios semióticos sirven para la recepción de un nuevo conocimiento solamente en manos de corifeos como Ch. Metz, U. Eco o R. Barthes. Pero en el desarrollo de toda ciencia se puede hablar de un período de estancamiento: During the past few years, this once elegant system of thought has produced little of real intellectual value $^{6}$.

Estas son diferentes aristas de un mismo fenómeno. Por un lado, la abundancia de metalenguajes, que dificulta la comprensión recíproca en el marco de una sola ciencia. Por otro lado, la hiperexplotación de uno o dos metalenguajes a los que se traducen los resultados de todos los análisis, y esta misma traducción al metalenguaje semiótico crea la ilusión de un nuevo conocimiento, produce un cientificismo que lleva incluso a resultados triviales. El excesivo caos metalingüístico también dificulta el desarrollo y la transformación del metalenguaje en una lengua prestigiosa que determina la percepción ${ }^{7}$.

Para valorar de una forma más correcta las propiedades ontológicas del objeto estudiado se debe distinguir, si no de una forma linguística sí metodológica, el objeto, su comprensión por medio del metalenguaje y la comprensión del propio metalenguaje; es decir, se debe distinguir en el análisis lo que emana de las propiedades del objeto estudiado y de las propiedades del metalenguaje.

Una posibilidad para esto es la así llamada traducción inversa del metalenguaje a la lengua objeto, la salida del sistema metalingüístico autosatisfactorio. Un buen ejemplo puede ser el semiótico polaco J. Pelc, que intentó comprender el sistema de $\mathrm{Ch}$. Peirce precisamente fuera del metalenguaje de Peirce, conocer su pensamiento real: $I$ wish to find out what he actually had in mind. I therefore ask questions. And I would very much like to hear competent answers to these questions,

5 Monaco, J. How to Read a Film. New York, Oxford: Oxford University Press, 1981,340 .

6 Monaco, J. Op. cit., 340. 495-508. 
but answers that are not formulated according to the rules of Peirce's sytle and poetics which his followers and commentators sometimes adopt as their own ${ }^{8}$.

Otra posibilidad surge del principio de la complementariedad: diferentes metalenguajes pueden estar próximos en los límites de una metodología interdisciplinar, es decir, tiene lugar una cierta traducción metodológica o una traducción a una metodología única. Esto significa un pensamiento relativamente preciso en una lengua imprecisa, compuesta por retazos de diferentes metalenguajes. La traducción metodológica es para la teoría de la traducción, al parecer, una posibilidad de salida del estancamiento. Al reconocer como objeto principal del estudio de la teoría de la traducción el proceso de la traducción, quisiéramos desarrollar la idea de I. Revzin y V. Rosenzveig de que «los tipos de realización del proceso de la traducción, incluida el de la traducción precisa, son únicos para todos los tipos de textos» ${ }^{9}$.

R. Jakobson parte en primer lugar de la lingüística cuando distingue, en su ya clásico trabajo, la traducción intralingüística (interpretación de los signos verbales mediante otros signos de esa misma lengua), la traducción interlingüística (interpretación de los signos verbales de una lengua mediante los signos de otra lengua) y la traducción intersemiótica o transmutación (interpretación de los signos verbales mediante sistemas sígnicos no verbales) ${ }^{10}$.

Desde el punto de vista del desarrollo de la teoría de la traducción parece más productivo la distinción entre traducción intratextual, textual y extratextual. La traducción intratextual significa una traducción completa o parcial de un texto a otro texto, y generalmente esta problemática de la palabra ajena, del texto en el texto, del intexto o intertexto, se estudia en el marco del análisis histórico-literario ${ }^{11}$. La traducción textual es la traducción en el sentido tradicional de la palabra, mientras que la traducción extratextual es la traducción de un texto, de un tipo de arte a otro (de la literatura al cine o al teatro, etc.), es decir,

8 Pelc, J. «Several Questions to Experts in Peirce's Theory of Signs». Versus 1990, n. ${ }^{\circ} 55 / 56,14$.

9 Revzin, I., Rozentsveig, V. Osnovy obschego i mashinnogo perevoda. Moscú, 1964, 173. Compárese: Torop, P., «Protsess perevoda i nekotorye metodologuicheskie problemy perevodovevedenia». Trudy po znakovym sistemam. XV. Tartu, 1982, 10-23.

10 Jakobson, R. «On Linguistic Aspects of Translation». Selected Writings. 2. Word and Language. The Hague, Paris: Mouton, 1971, 261.

11 Sobre este aspecto de la traducción véase: Torop P. «Problema inteksta», Trudy po znakovym sistemam. XIV. Tartu, 1981, 33-44. 
el texto se saca de sí mismo. La traducción extratextual generalmente se estudia en los límites de la semiótica de Peirce ${ }^{12} \mathrm{o}$ de los estudios fílmicos o teatrales ${ }^{13}$.

Un mismo planteamiento metodológico ante problemas aparentemente tan diferentes descubre en mayor medida la ontología del texto, en general, y la ontología del texto traducido como un tipo especial de texto, en particular. Aparte de esto, partiendo del modelo teórico del proceso de la traducción se puede establecer, por ejemplo, una tipología, comparar diferentes tipos de adaptación cinematográfica, así como describir las posibilidades esenciales de la adaptación de un texto literario. Al mismo tiempo, el análisis de los filmes permite mirar de forma un poco diferente la traducción escrita.

La traducción metodológica permite también introducir, productivamente y sin dificultad, principios del análisis semiótico en la teoría de la traducción. Generalmente se mencionaba a la semiótica, ante todo, en relación con los problemas histórico-literarios de la traducción, con el reconocimiento de los signos culturales. En los últimos tiempos, la situación cambia un poco y la semiótica se considera útil para el análisis de la transmisión en la traducción de una comunicación no verbal, así como para el estudio de la interrelación entre el lector y el texto de la traducción ${ }^{14}$.

En los últimos tiempos, la atención ha pasado de la cultura de partida a la cultura de recepción. Incluso desde el punto de vista de la teleología de la traducción, G. Toury considera que la traducción no es la comunicación de un mensaje verbal a través de una barrera lingüísticocultural, sino la comunicación en un mensaje traducido dentro de un determinado sistema lingüístico-cultural (communication in translated messages within a certain cultural-linguistic system ${ }^{15}$. Pero acentuar la dialoguicidad en las relaciones entre el texto y el lector conduce de la codificación de la percepción por el propio texto a una determinación cultural. El planteamiento comunicativo enmascara la ontología del texto.

12 Plaza, J. Tradução intersemiótica. São Paulo, 1987, 89-93.

13 Compárese este aspecto de la traducción: Torop, P. «Kirjandus ja film», Teater. Muusika. Kino 1987, n. ${ }^{\circ} 1,16-25$; P. Torop. «Literatura i film», Kinovedcheskie zapiski 1989, n. $^{\circ}$ 5, 9-24.

14 Gentile, A. «The Application of Theoretical Constructs from a Number of Disciplines for the Development of a Methodology of Teaching in Interpreting and Translating», Meta 1991, vol. XXXVI, n. ${ }^{\circ} 2 / 3,347$.

15 Toury, G. In Search of a Theory of Translation. Tel Aviv: The Porter Institute for Poetics and Semiotics, 1980, 17. 
Al mismo tiempo, es preciso reflexionar sobre la idea, a primera vista paradójica, de A. Kasher de que no existen pruebas contundentes para considerar que la lengua natural, en su esencia, sea un sistema de comunicación: Often, subscription to the view that natural language is in essence a system of communication is nothing more than an expression of some pre-theoretic, uncritical intuition ${ }^{16}$.

La competencia lingüística es posible también sin una competencia cultural cuando la comunicación no posee un fin especial ${ }^{17}$, es decir, cuando el texto en la lengua ajena y el texto en la lengua nativa pueden ser percibidos de forma idéntica. Esto se puede ilustrar con la adaptación cinematográfica de la literatura. Por un lado, el texto literario se desmorona en partes, unidas después en un único filme. Parte del libro se conserva en la lengua natural en los diálogos de los personajes, parte de las descripciones se convierten en visuales, las reflexiones del autor las puede transmitir un locutor situado fuera de la escena, las emociones del autor pueden ser expresadas mediante el color, la luz, el escorzo y la música, el montaje transmite la trama. Por otro lado, puede compararse la adaptación cinematográfica con la lectura de un libro, donde los personajes hablan en una lengua, las descripciones de la naturaleza se dan en otra lengua, las reflexiones del autor en una tercera, etc. El problema del envejecimiento de las traducciones demuestra que la unidad lingüística de la traducción es incomparablemente menor que la del original. El lector de la traducción está cercano al espectador de la adaptación. Si la traducción o la película estimula la imaginación, libera los canales de la percepción, creando una unidad visual-acústica, la lengua propiamente dicha pasa a un segundo plano. Esto significa que la coherencia en la traducción se consigue no solamente en el nivel de la lengua. Antes de esto es imprescindible una unidad visual, lo que se actualiza en la adaptación.

Sobre la unidad visual escriben los propios traductores y directores de cine, no solamente los investigadores. Lo principal en el trabajo del traductor se considera la representación visual del texto, incluso antes de empezar el propio trabajo de la traducción ${ }^{18}$ y consecuentemente

16 Kasher, A. «On the Pragmatic Modules: a Lecture». Journal of Pragmatics 1991, vol. $16, \mathrm{n}^{\circ} 1,383$. 174.

Agar, M. «The Biculture in Bilingual». Language in Society 1991, vol. 20, n. 2 ,

18 Schulte, R. «Translation: An Interpretative Act through Visualization». Translational Agent of Communication. A Special issue of «Pacific Moana Quarterly»1980, vol. $5, \mathrm{n} .^{\circ} 1,82$. 
se considera un gran peligro de la traducción la desaparición de la representación visual del texto ${ }^{19}$.

En el marco del cronotopo, lo visual está estrechamente relacionado con lo temporal y, en el plano de la coherencia lingüística de la traducción, se puede afirmar que en la traducción esta frecuente ausencia de unidad en la utilización del tiempo gramatical surge en muchos casos de la incapacidad de la representación visual del texto, de la comprensión de los cronotopos del texto.

Los análisis demuestran que incluso el lector que no puede valorar la calidad lingüística de la traducción, es capaz de distinguir, sin embargo, una buena o mala traducción. Por ejemplo, en Georgia, dieron a leer a dos grupos diferentes de personas, georgianos que dominaban el ruso y rusos que no dominaban el georgiano, fragmentos de nueve traducciones de Merani de Baratashvili y seis traducciones de $E l$ guerrero con piel de tigre. Ambos grupos fueron unánimes en la distinción de la peor traducción y estuvieron muy cercanos en la definición de la mejor. A los rusos les gustaba más El guerrero..., de Zabalotski; en segundo lugar, pusieron la traducción de Petrienko, y los georgianos al revés. Ambos grupos no supieron explicar por qué no les gustaba la traducción de Pasternak ${ }^{20}$.

Por experiencia propia puedo añadir la actitud de los lectores hacia las nuevas redacciones de traducciones que se han quedado anticuadas por su lengua. La renovación del texto, tan sólo desde el punto de vista de la redacción, viola tanto la unidad interna del texto que muchos lectores prefieren las traducciones antiguas pero internamente más unidas.

Por supuesto, la visualidad y, en un sentido más amplio, la perceptibilidad difícilmente se someten a análisis. Por ejemplo, una traductora estonia de la literatura francesa llego por primera vez a París a una edad ya avanzada. Pero al volver dijo que para la actividad traductora el viaje había dado poca cosa, ella recordaba solamente algunos olores.

Ejemplos parecidos demuestran que en la actividad traductora, tanto en la etapa de engendramiento como en la de percepción del texto, más importante que el logro de la unidad lingüística es el de la unidad perceptiva. La unidad perceptiva puede lograrse en el nivel de

19 Caws, M. A. «Literal or Liberal: Translating Perception». Critical Inquiry 1986, vol. $13, \mathrm{n}^{\circ} 1,60-61$.

20 Dzharsheishvili R. Psijologuicheskaia problema judozhestvennogo perevoda. Tbilisi: Metsiereba, 1984, 54-60. 
la lengua y también mediante comentarios dentro o fuera del texto de la traducción. El texto puede ser plurilingüe, en el sentido lingüístico o semiótico, pero ese plurilingüismo debe subordinarse no a leyes de coherencia linguística sino a las de coherencia semiótica del texto, sea ésta la utilización del ruso y del francés en Guerra y paz, de Tolstoi, la mezcla del inglés y el francés en la literatura canadiense o la adaptación cinematográfica de cualquier texto literario.

Si el director de la adaptación está obligado simplemente a poner el texto literario en partes transmisibles y perceptibles por diferentes canales, en la actividad traductora tan sólo ahora se empieza a tener conciencia de que el texto literario se comunica con el lector no sólo por medio de la lengua. $Y$ el traductor tiene la posibilidad, $y$ con frecuencia la necesidad, de poner el texto en partes para comprender el plurilingüismo semiótico del texto como coexistencia y no como mezcla de lenguas. En el sentido semiótico, la traducción de un texto siempre significa una traducción simultánea de unas cuantas lenguas, y la principal cuestión consiste en cómo conservar la coherencia sin mezclar estas lenguas ${ }^{21}$.

Es inaccesible para el traductor el sincretismo del autor del original, él como director de la adaptación está obligado a concretar lo inconcreto y a explicitar lo implícito, a comprender las lenguas del texto por separado para unirlas de nuevo en la traducción. Para esto, junto con la estructura del texto, se debe estudiar la estructura del mundo en el texto, la relación de los cronotopos de la imagen de la realidad que tiene el autor, de la concepción artística de la obra y del cronotopo del personaje. Su simultaneidad y, al mismo tiempo, su distinción crean también la base imprescindible para el análisis de la traducción y para la comprensión de la técnica que permite alcanzar la coherencia, incluso en una situación de violación del sincretismo del original y de la explicitación del plurilingüismo semiótico.

21 Compárese esto con el llamamiento a conservar la pureza de las lenguas en una sociedad plurilingüe: Mezei, K. «Speaking White. Literary Translation as a Vehicle of Assimilation in Quebec». Canadian Literature 1988, 11-23. 